\title{
Task-generic and task-specific connectivity modulations in the ADHD brain: an integrated analysis across multiple tasks
}

\author{
Roselyne J. Chauvin (10 ${ }^{1,2,3}$, Jan K. Buitelaar ${ }^{1,2,4}$, Emma Sprooten (10 ${ }^{1,2}$, Marianne Oldehinkel ${ }^{1,2,5}$, Barbara Franke $\mathbb{C}^{2,6}$, \\ Catharina Hartman ${ }^{7}$, Dirk J. Heslenfeld ${ }^{8}$, Pieter J. Hoekstra', Jaap Oosterlaan ${ }^{8,9}$, Christian F. Beckmann ${ }^{1,2,10}$ and \\ Maarten Mennes ${ }^{2}$
}

\begin{abstract}
Attention-deficit/hyperactivity disorder (ADHD) is associated with altered functioning in multiple cognitive domains and neural networks. This paper offers an overarching biological perspective across these. We applied a novel strategy that extracts functional connectivity modulations in the brain across one $\left(P_{\text {single }}\right)$, two $\left(P_{\text {mix }}\right)$ or three $\left(P_{\text {all }}\right)$ cognitive tasks and compared the pattern of modulations between participants with ADHD $(n-89)$, unaffected siblings $(n=93)$ and controls ( $n=84$; total $N=266$; age range $=8-27$ years). Participants with ADHD had significantly fewer $P_{\text {all }}$ connections (modulated regardless of task), but significantly more task-specific $\left(P_{\text {single }}\right)$ connectivity modulations than the other groups. The amplitude of these $P_{\text {single }}$ modulations was significantly higher in ADHD. Unaffected siblings showed a similar degree of $P_{\text {all }}$ connectivity modulation as controls but a similar degree of $P_{\text {single }}$ connectivity modulation as ADHD probands. $P_{\text {all }}$ connections were strongly reproducible at the individual level in controls, but showed marked heterogeneity in both participants with ADHD and unaffected siblings. The pattern of reduced taskgeneric and increased task-specific connectivity modulations in ADHD may be interpreted as reflecting a less efficient functional brain architecture due to a reduction in the ability to generalise processing pathways across multiple cognitive domains. The higher amplitude of unique task-specific connectivity modulations in ADHD may index a more "effortful" coping strategy. Unaffected siblings displayed a task connectivity profile in between that of controls and ADHD probands, supporting an endophenotype view. Our approach provides a new perspective on the core neural underpinnings of ADHD.
\end{abstract}

\section{Introduction}

Attention-deficit/hyperactivity disorder (ADHD) is a mostly early onset neurodevelopmental disorder characterised by symptoms of inattention and/or hyperactivity-impulsivity that are associated with impairments in multiple functional domains ${ }^{1}$. Multiple cognitive theories have been proposed to explain the underlying

\footnotetext{
Correspondence: Roselyne J. Chauvin (chauvin@wustl.edu)

'Department of Cognitive Neuroscience, Radboud University Medical Center, Nijmegen, The Netherlands

2Donders Institute for Brain, Cognition and Behaviour, Radboud University, Nijmegen, The Netherlands
}

Full list of author information is available at the end of the article core deficits of the disorder, including a dysfunction in state and arousal regulation ${ }^{2}$, response inhibition ${ }^{3}$, broader executive functioning ${ }^{4}$, motivation ${ }^{5}$ and/or delay aversion $^{4}$. Functional imaging studies building on these cognitive explanations have investigated the neural underpinnings of ADHD, but have revealed a heterogeneous pattern of altered neuronal function spread across the brain ${ }^{1,6-8}$. This fragmented pattern of findings asks for new integrated approaches that provide an overarching perspective on the functional architecture of the ADHD brain across cognitive domains.

Here, we offer such a perspective by applying a novel approach that integrates findings of cognitive tasks across 
multiple cognitive domains to assess the role of taskdependent connectivity modulations ${ }^{9}$. We thereby capitalize on the idea that task-induced connectivity patterns build on the baseline functional connectivity architecture as indexed by resting-state MRI analyses ${ }^{10-12}$. Using the resting-state architecture as baseline allows assessment of the regional specifics and magnitude of task-induced connectivity modulations across task paradigms ${ }^{9}$. Specifically, we use task potency as a metric to index the strength of task-induced connectivity modulations in terms of their difference from a resting-state baseline. This approach facilitates the comparison between task paradigms and permits to disentangle modulations that are shared across multiple cognitive functions (i.e. $P_{\text {all }}$ ), resembling a cognitive core ${ }^{13-15}$, from those that are taskspecific (i.e. $P_{\text {single }}$ ). For example, a comparison of working memory, response inhibition and reward tasks could reveal that participants with ADHD show alterations in the same network of inhibition-related brain regions in each task. This would provide support for theories that claim a prominent role for poor response inhibition in $\mathrm{ADHD}^{3}$. Alternatively, theories suggesting inefficient management of resources in participants with ADHD would be supported by observing, for example, a pattern of modulations that is highly specific to each task, while an overall cognitive core in support of task-general processes remains under-modulated ${ }^{16,17}$. Both theories are not incompatible: an alteration in one function network may induce coping strategies involving other functional networks. In combination, this may manifest as inefficiency across multiple task-generic and task-specific systems. In light of these possibilities we hypothesized that, in ADHD, the brain's functional core interacts differently with more specialized network modulations, which could reflect inefficient use of the brain's resources in ADHD.

To test our hypothesis, we applied our task-potency framework to a large cohort of participants with ADHD, their unaffected siblings, and healthy controls $(N=266)$ and describe functional connectivity patterns across three cognitive domains (probing response inhibition ${ }^{16}$, working memory ${ }^{18}$ and reward processing ${ }^{17}$ ). Since unaffected siblings share on average $50 \%$ of genetic variation with their ADHD probands, the addition of unaffected siblings allowed assessing the impact of familial vulnerabilityaddressing the hypothesis that unaffected siblings show a task connectivity modulation profile that is an intermediate phenotype between that of diagnosed siblings and control participants ${ }^{19,20}$.

\section{Methods}

\section{Participants and (f)MRI acquisitions}

We selected participants with ADHD, unaffected siblings of individuals with ADHD (but not related to the participants with ADHD included in this study), and typically developing controls (unrelated to any participant) from the NeuroIMAGE sample ${ }^{21}$. All selected participants completed an anatomical MRI scan, a resting state fMRI scan (RS), and at least one of the following task fMRI scans: a spatial working memory task (WM), a monetary-incentive-delay reward task (REWARD) and/or a stop signal response inhibition task (STOP) (see Table S1). Table 1 summarizes the demographics of the 89 participants with ADHD, 93 unaffected siblings, and 84 controls included in the current analyses. A full description of the selection criteria, task paradigms and MRI acquisition parameters is provided in Table S2 and S3 and supplemental method (appendices). Participants were scanned at two different sites; therefore we provide, in supplementary Figs. 1 and 2, a replication of all results within site and with matched samples on age, IQ, site, gender.

\section{Task potency calculation}

Our task potency approach is described in detail elsewhere 9 . In brief, for each participant and each preprocessed RS, WM, REWARD and STOP fMRI acquisition (see eMethod for pre-processing procedures) we defined functional connectivity matrices using 179 regions from a hierarchical whole-brain atlas ${ }^{22}$ (see Fig. S3). We calculated connectivity as the normalized Fisher- $Z$ partial correlation between the timeseries of each pair of regions in the atlas. We used a mixture modelling approach ${ }^{23}$ for the normalization in order to correct for residual autocorrelation of each $\mathrm{Z}$ matrix. The central Gaussian fits the 'null' part of the matrix values and is used as normal reference (see Supplementary Method). To isolate connectivity changes induced by task modulation (WM, REWARD, STOP) from changes in the brain's baseline architecture (RS), we standardized each individual-level pair-wise correlation obtained during task acquisition by subtracting the corresponding pair-wise correlation value calculated for the RS scan of that participant. This effectively allows comparing each connection in the task connectivity matrices in terms of its magnitude of deviation from that participant's resting baseline ${ }^{9}$. We refer to this deviation as 'task potency', which is considered as an absolute value and disregards complex interpretation of down- or up-modulation.

For each task, we created group-level task potency matrices by averaging the individual-level potency matrices across all participants in each diagnostic group. Within these group-level matrices we selected those connections that were sensitive to task modulation, by thresholding each group-level task potency matrix. Negative and positive thresholds were defined using a two-tailed version ${ }^{9}$ of mixture modelling thresholding ${ }^{24,25}$. We used the most conservative limit across groups (as controls, ADHD, and Sibling groups were not equal size) in order to compare 
Table 1 Participant information: descriptive, clinical variables, head movement during scanning and distribution of scan modalities, for each group sample and tasks: resting state (RS), stop signal paradigm (STOP), reward processing (REWARD), working memory (WM).

\begin{tabular}{|c|c|c|c|c|c|c|c|c|c|}
\hline & $\begin{array}{l}\mathrm{N} \text { used in } \\
\text { final } \\
\text { analyses }\end{array}$ & $\begin{array}{l}\text { Age } \\
\min -\max \end{array}$ & $\begin{array}{l}\text { Age } \\
\text { mean (std) }\end{array}$ & $\%$ female ${ }^{* *}$ & Site $^{b}$ & Inattention $^{\mathrm{a}}$ (std) & Hyperactivity $^{\mathrm{a}}$ (std) & $\mathrm{IQ}^{* *}(\mathrm{std})$ & $\begin{array}{l}\text { Root mean } \\
\text { square head } \\
\text { movement (std) }\end{array}$ \\
\hline \multicolumn{10}{|c|}{ Healthy control participants } \\
\hline RS & 84 & $10.8-23.5$ & $16.7(3)$ & $50 \%$ & $65 \%$ & $0.4(1.1)$ & $0.3(0.8)$ & $107.2(13.8)$ & $0.14(0.17)$ \\
\hline STOP & 46 & $10.8-23.4$ & $16.7(3.1)$ & $58.7 \%$ & $50 \%$ & $0.3(1.0)$ & $0.2(0.6)$ & $106.1(14.1)$ & $0.10(0.06)$ \\
\hline REWARD & 46 & $12.7-23.5$ & $16.6(2.9)$ & $50 \%$ & $55 \%$ & $0.6(1.5)$ & $0.3(0.9)$ & $108.1(12.8)$ & $0.16(0.11)$ \\
\hline WM & 66 & $10.8-23.5$ & $16.5(3.1)$ & $50 \%$ & $80 \%$ & $0.3(1.0)$ & $0.3(0.8)$ & $105.8(13.4)$ & $0.18(0.16)$ \\
\hline \multicolumn{10}{|c|}{ Unaffected Siblings } \\
\hline RS & 93 & $7.7-28.1$ & $16.9(4)$ & $57 \%$ & $45 \%$ & $0.7(1.4)$ & $0.6(1.1)$ & $101.6(14.9)$ & $0.16(0.21)$ \\
\hline STOP & 55 & $7.7-27.3$ & $17.1(4.1)$ & $54.5 \%$ & $36 \%$ & $0.7(1.7)$ & $0.7(1.3)$ & $102.6(16.3)$ & $0.10(0.10)$ \\
\hline REWARD & 57 & $9.1-28.1$ & $17.2(3.7)$ & $57.9 \%$ & $49 \%$ & $0.6(1.4)$ & $0.7(1.2)$ & $100.1(15.2)$ & $0.11(0.07)$ \\
\hline WM & 44 & $7.7-28.1$ & $16.2(4.1)$ & $56.8 \%$ & $54 \%$ & $0.3(1.0)$ & $0.3(0.7)$ & $102.6(13.7)$ & $0.15(0.21)$ \\
\hline \multicolumn{10}{|c|}{ ADHD participants } \\
\hline RS & 89 & $8.5-24.5$ & $17.4(3.1)$ & $31.5 \%$ & $38 \%$ & $7.3(1.9)^{*}$ & $5.8(2.3)^{*}$ & $94.4(14.5)$ & $0.24(0.38)$ \\
\hline STOP & 49 & $11.1-24.5$ & $17.7(2.7)$ & $22.4 \%$ & $37 \%$ & $7.3(1.5)^{*}$ & $5.3(2.3)^{*}$ & $95.8(14.7)$ & $0.09(0.05)$ \\
\hline REWARD & 57 & $10.2-24.5$ & $17.7(3.1)$ & $35.1 \%$ & $32 \%$ & $7.0(1.5)^{*}$ & $6.1(2.0)^{*}$ & $97.0(14.9)$ & $0.17(0.20)$ \\
\hline WM & 51 & $10.2-24.2$ & $17.2(3.23)$ & $35.3 \%$ & $47 \%$ & $7.5(1.3)^{*}$ & $5.8(2.3)^{*}$ & $94.6(14.3)$ & $0.24(0.21)$ \\
\hline
\end{tabular}

Replication of analysis for differences in sample (scanner/site, gender, IQ) is available in Figs. S1 and S2.

For participant exclusion, see Table S6. **For more detail on IQ and gender representation and testing of differences, see Fig. S7.

${ }^{a}$ Combined symptoms from KSADS and Conners.

${ }^{\mathrm{b}}$ Ratio of Amsterdam/Nijmegen scan localisation.

the same level of information and selected potency values exceeding this limit as being sensitive to task modulation.

To integrate results across task-paradigms, we subdivided these sensitive connections depending on their modulation by one or more of the tasks. In particular, we refer to connections that were modulated by one task only as $P_{\text {single }}$, to connections that were modulated by more than one but not all tasks as $P_{\text {mix }}$, and to connections that were modulated regardless of task as $P_{\text {all }}$. We verified that the relative percentage of participants that performed multiple acquisitions was equivalent between groups to avoid a possible bias related to participant by task interactions in edge selection (see details in Supplementary Table 1).

\section{Group differences in task connection type}

To assess whether ADHD was associated with a deviant distribution of task-induced modulations across the brain and across tasks, we compared the distribution of tasksensitive connections and relative $P_{\text {single, }}, P_{\text {mix }}$ and $P_{\text {all }}$ connections across the three diagnostic groups.

We compared the amount of sensitive connections between groups by indexing the percentage of connections included for each group relative to the total number of sensitive connections. We assessed between-group differences in the specificity of connections by obtaining for each group the percentage of connections per type relative to the total number of sensitive connections for that group. Finally, we assessed the ratio of connections uniquely modulated by each diagnostic group (unique connections) versus those connections that were also modulated by one or both of the other groups (shared connections).

To account for the heterogeneity in the population, we used a bootstrapping procedure to statistically infer group differences against an appropriate null distribution. Defining empirical null distributions specifically for each diagnostic group allows controlling for sensitivitydifferences due to relative group sizes across tasks and across diagnosis. We sub-selected $80 \%$ of the study population, computed the different metrics of interest, i.e. true values (percentage of connections per label and amplitude of modulation), randomly relabelled the participants keeping group size equal and computed the same values for a random expectation. We perform this subselection and procedure 10,000 times in order to build a 
null distribution. We tested for significant differences between the average observed values across 10,000 subselections and the obtained null distribution (for further details see Supplementary Methods).

Using this procedure, we tested differences in the percentage of connections modulated across tasks (tasksensitive, $P_{\text {all }}, P_{\text {mix }}, P_{\text {single }}$ connections), and in the percentage of shared versus unique connections for each of the connection labels. To further assess whether differences in percentage of selected connections were associated with different amounts of modulation, we compare their average amplitude of modulation and tested values against the corresponding diagnostic group specific null distribution. $P$ values were assessed for significance using FDR correction across tests per group at $q<0.05$. Replication of values in light of possible confounder effects (medication, gender, scanner of acquisition and comorbidity) are presented in Fig. S4.

Finally, we assessed the stability versus the heterogeneity of task connection types in the different groups. To this end we used the 10,000 extracted values from the $80 \%$ sample bootstrapping procedure and computed each connection's selection rate at the group level across bootstraps and its associated shared selection rate between two groups. We computed these rates for sensitive, $P_{\text {all }}, P_{\text {mix }}$ and $P_{\text {single }}$ connections. These group-level selection rates index how specific a selected connection is to one particular group by computing the difference in selection rate between groups for each connection. We can then display the uniqueness (for a specific group) versus the sharedness (across groups) of each connection. By comparing both rates, we can estimate which connections are uniquely and reproducibly selected in one group only, potentially representing idiosyncratic strategies to solve a task.

\section{Results}

\section{Establishing an ADHD connectivity profile}

Starting from the set of connections that yielded significant connectivity modulations across all participants, we compared the diagnostic groups in terms of the sensitivity and specificity of their connectome to task modulations. Figure 1 shows that participants with ADHD modulated a significantly smaller part of sensitive connections (mean $40.1 \%$ of all sensitive edges, $\mathrm{SD}=4.7$, $P<0.005)$ compared with the group-specific null distribution $(50.6 \%, \mathrm{SD}=4.1)$, while the percentage of edges modulated by controls and siblings was not significantly different from random expectation $(47.4 \%, \mathrm{SD}=4.4$ and $44.5 \%, \mathrm{SD}=4.5$, respectively).

Illustrating the task-specific nature of the sensitive connections, the bottom part of Fig. 1 displays the proportion of selected connections that were $P_{\text {all }}$ or $P_{\text {single }}$ (full results including $P_{\text {mix }}$ edges are available in Fig. S4).
ADHD had $13.8 \% P_{\text {all }}$ modulations, which was significantly lower than expected $(P=0.031)$, and lower compared to both controls (17.8\%) and siblings (16.3\%). Within the $\mathrm{P}_{\text {all }}$ connections, ADHD seemed to mostly modulate connections that controls and siblings also modulated (the percentage of shared connections was $>10 \%$ higher compared with the other groups, yet, this group difference did not reach significance).

In contrast to the lower number of $P_{\text {all }}$ connections, the ADHD group exhibited a significantly higher percentage of $P_{\text {single }}$ connections compared with the null distribution (see Fig. 1). While the sibling group also showed a high percentage of $P_{\text {single }}$ connections, this difference did not survive multiple comparison corrections across tests within this group. We observed no significant betweengroup differences in the uniqueness of the $P_{\text {single }}$ connections.

\section{Reproducibility-from group level to individual analyses}

We assessed the variability of the task connection types across bootstraps within each group, to examine the homogeneity of results across participants within a group. Figure 2 shows the results of these analyses for $P_{\text {all }}$ and $P_{\text {single }}$ edges (task-sensitive and $P_{\text {mix }}$ related results can be found in Fig. S5). The $P_{\text {all }}$ connections in particular displayed strong homogeneity across control participants, with a notable shift toward $100 \%$ selected for controls, demonstrating a high reproducibility in controls and missing characterisation of these edges as $P_{\text {all }}$ in other groups. In other words, controls reliably modulated $P_{\text {all }}$ connections that were not consistently modulated by both other groups. In contrast, siblings seemed to modulate an alternative set of $P_{\text {all }}$ connections that were not used by controls (Fig. 2 top right). This further informs on differences and similarities observed in Fig. 1. In contrast, the $P_{\text {single }}$ connections observed in ADHD and siblings (see Fig. 1) were heterogeneous across participants, as illustrated by an absence of a shift in the distributions shown in Fig. 2 towards the ADHD and sibling groups (see also Fig. S5). Finally, we refer to Fig. 3 and S6 for a description of the location of $P_{\text {all }}$ connections that were strongly selected in each of the diagnostic groups.

\section{Amplitude of modulation}

As a proxy for cost estimation of different connectivity profiles reflecting possible compensatory mechanisms, we assessed whether the group differences in connectivity profiles were associated with group differences in the amplitude of the modulations. Figure 4 shows that siblings and controls equally modulated the different connection types (Table S4 provides statistical details). However, participants with ADHD overmodulated connections that were unique to them. This overmodulation was significant for $P_{\text {single }}$ connections and $P_{\text {mix }}$ 


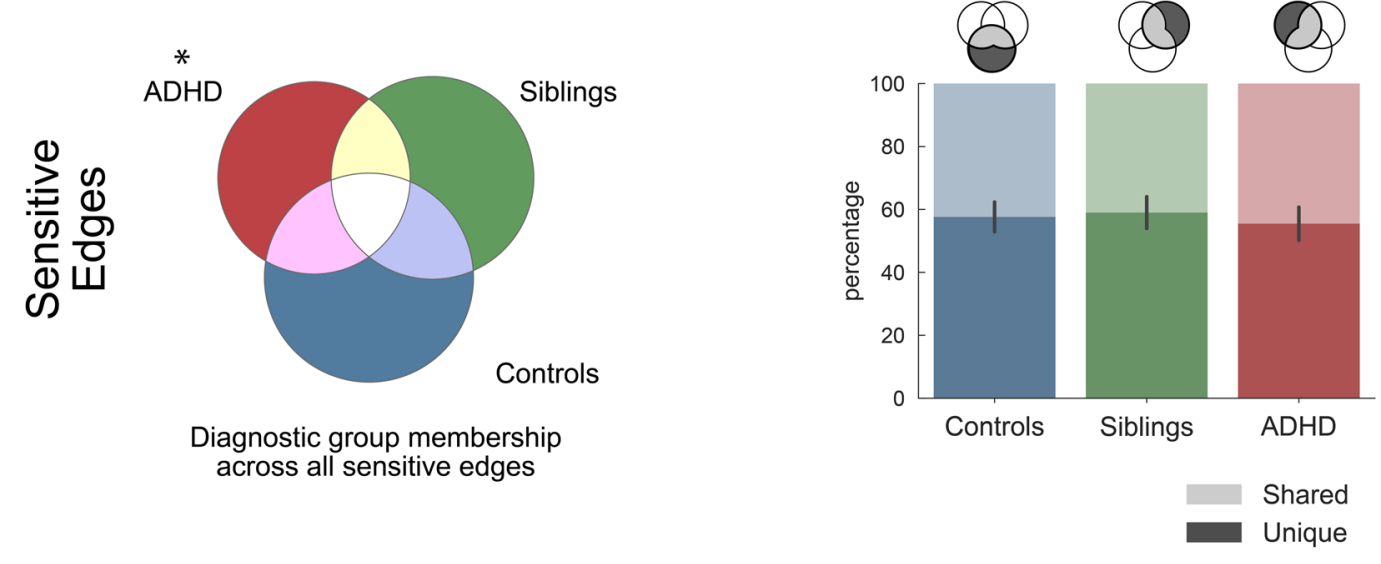

\section{Specificity of sensitive edges}
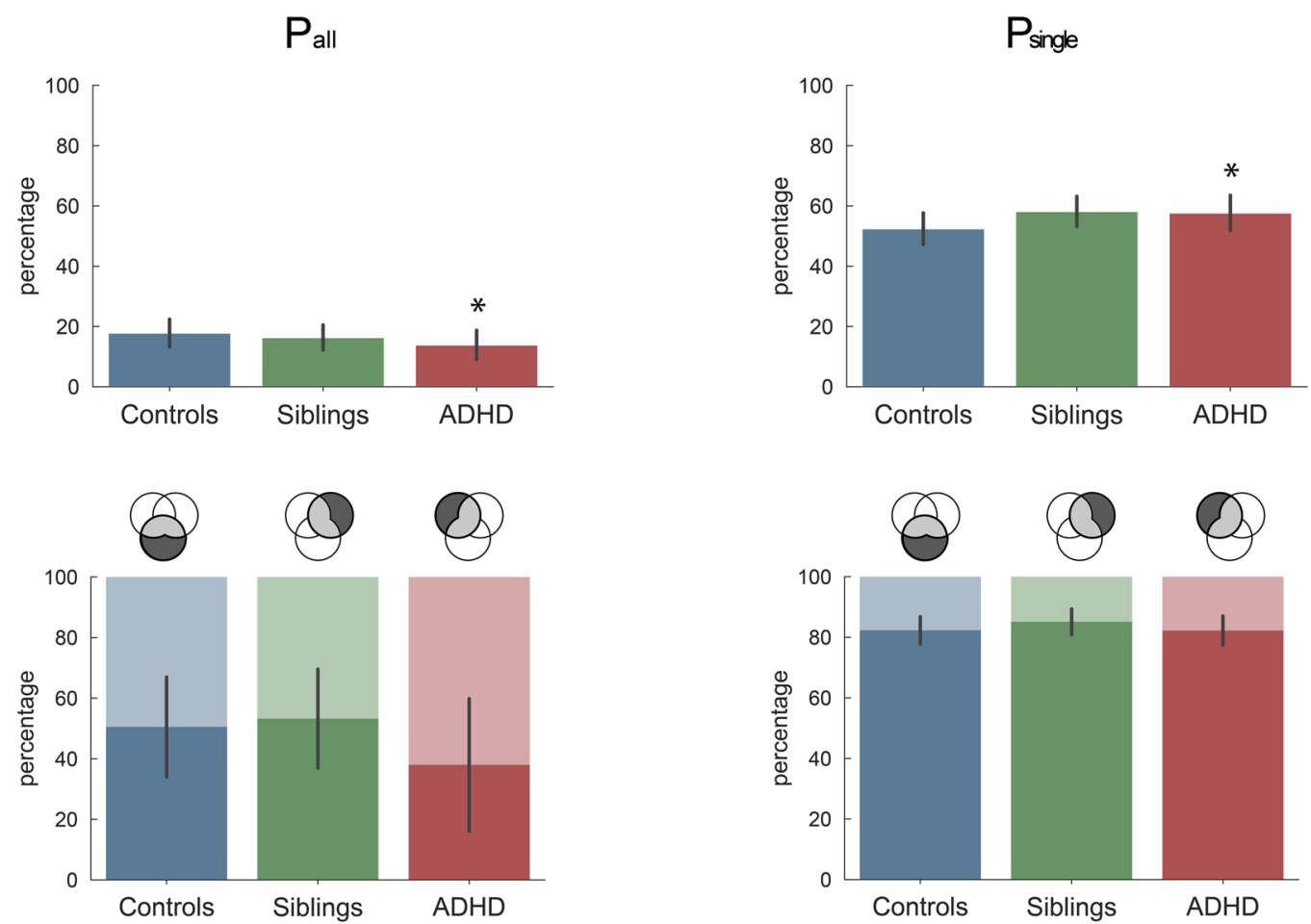

Fig. 1 Sensitivity to task modulation. Description of total connectivity modulations across the three tasks and diagnostic groups (ADHD, siblings, controls). The first row shows for each group the percentage of connections they modulated across the three tasks (sensitive connections) and within these selected connections, the relative percentage of connections unique to one group or shared across groups. We further split the selected connections of each group into task-specific $\left(P_{\text {single }}\right)$, and common $\left(P_{\text {all }}\right)$ connections, corresponding to connections modulated in only one, or all three tasks, respectively. The second row of this figure quantifies the relative percentage of each connection type $\left(P_{\text {all }}\right.$ and $\left.P_{\text {single }}\right)$ within the sensitive connections of each group. For the connections described in the second row, the third row then quantifies whether these connections were unique to that group or shared across groups. Stars indicate significant differences from null distribution after FDR correction (see Table S7). Replication of these findings across possible confounding effects (scanner, gender, medication, comorbidity, age, IQ) is available in Figs. S1 and S2.

connections (see Table S4), but did not reach significance for $P_{\text {all }}$ connections. This is in line with the idea that the ADHD group placed more emphasis on $P_{\text {single }}$ and $P_{\text {mix }}$ rather than on generic connections.

\section{Confounder effects}

In Supplementary Figs. 1 and 2, we replicated Figs. 1 and 3 to investigate age, IQ, gender, comorbidity, scan site and medication effect with matched samples. The new 


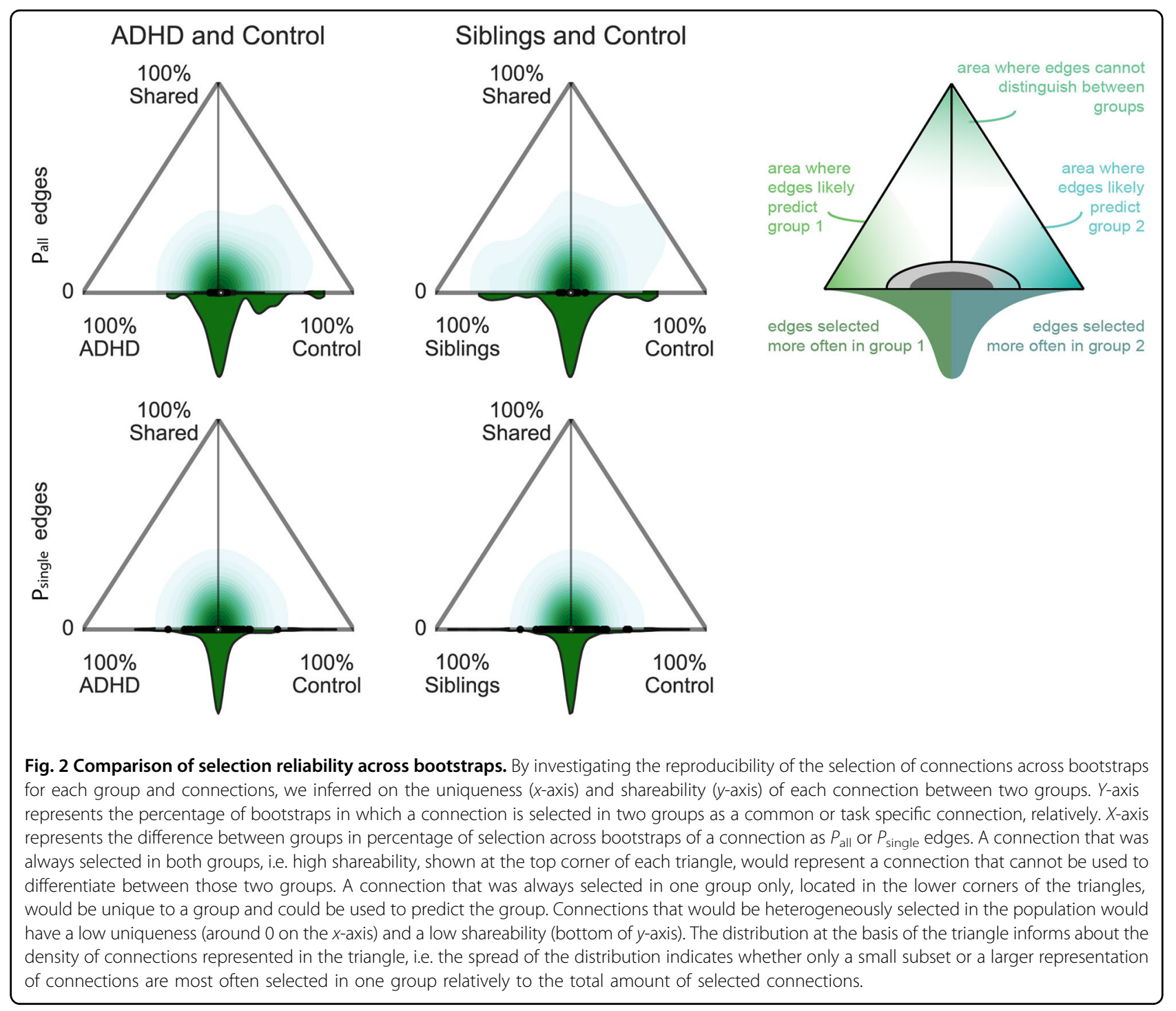

sample size being reduced, we did not perform the bootstrapping procedure. We observed an overall replication of effects with a lower $P_{\text {all }}$ and higher $P_{\text {single }}$ percentage in ADHD and a partial similarity of connectivity profiles between unaffected siblings and ADHD groups.

\section{Discussion}

We used a novel framework to provide an overarching perspective on the neurobiology of ADHD by inferring the nature of connectivity modulations in ADHD under the demands of working memory, reward processing, and response inhibition tasks. Our framework reveals that participants with ADHD activate significantly fewer connections than expected to perform each task. Furthermore, they modulated significantly fewer task-generic connections that share resources across tasks, and instead, relied significantly more on unique sets of connections $\left(P_{\text {single }}\right)$. In turn, participants with ADHD over-modulated those $P_{\text {single }}$ connections, suggesting a task-tailored potential compensatory mechanism. In comparison, unaffected siblings of ADHD participants displayed an intermediate phenotype with values in between those observed for controls and ADHD.

The ADHD population is known for its clinical, biological and etiologic heterogeneity, and it is possible that different etiologic and/or biological mechanisms could result in the same behavioural symptoms. The results of our group-based analyses together with the high reproducibility at the individual level strongly support the idea that a core alteration underlies the cognitive and neural impairments observed in ADHD. Participants with ADHD rely less on a $P_{\text {all }}$ core of modulations and instead involve task-tailored patterns of connectivity. These observations further support the idea that ADHD is characterized by neural inflexibility ${ }^{26,27}$, as the use of predominantly task-tailored connectivity patterns makes 
Areas with most reliable Pall connections used in both groups (ratio difference $<25 \%$ )

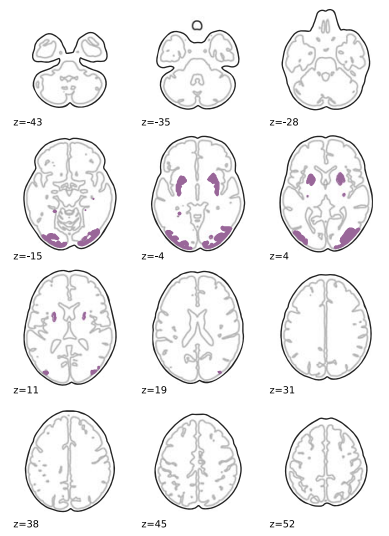

Most reliable Pall connections in $\mathrm{ADHD}$ vs Controls

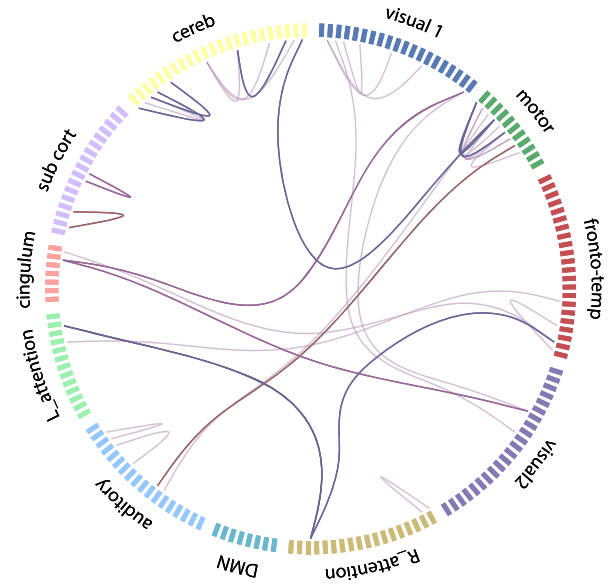

selection $>25 \%$ and $\begin{aligned} & \text { ratio difference }>25 \% \text { : used more often }- \text { in Controls } \quad \text { in ADHD } \\ & \text { ratio difference }<25 \%\end{aligned}$
Areas with most reliable Pall connections used more often in one or the other group

selection $>5 \%$

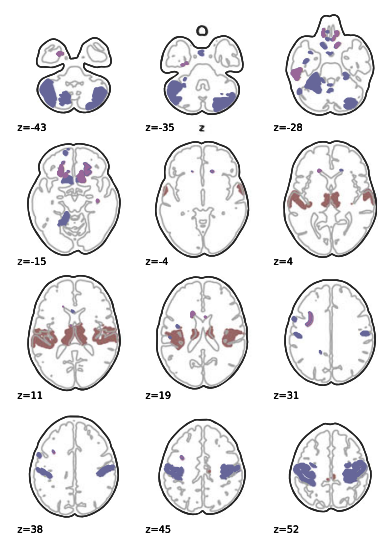

Fig. 3 Representation of areas with most reproducible $P_{\text {all }}$ connections comparing ADHD and Control groups. The brain slices on the left show areas with at least one connection used reproducibly ( $>50 \%$ of selection and ratio difference $<25 \%$ ) in both groups. The circular connectivity plot represents all connections selected $>50 \%$ as $P_{\text {all }}$ in one of the two compared groups across bootstraps. Within these strongly reproducible $P_{\text {all }}$ connections, the ones used most often in one group are represented with full color of that group (ratio difference $>25 \%$ ) (red for ADHD, blue for Controls). The brain slices on the right represent the brain areas associated to the connections used more often in one group. If an area has connections used in each group, its color is a blend of the compared group colors (purple).

\section{$P_{\text {all }}$}

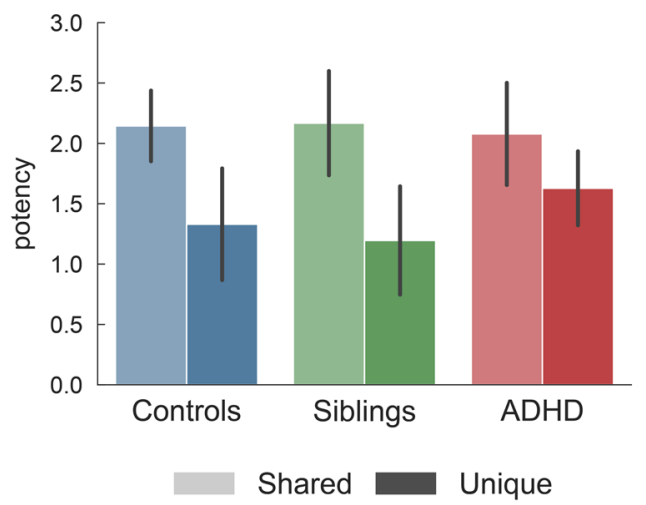

$P_{\text {single }}$

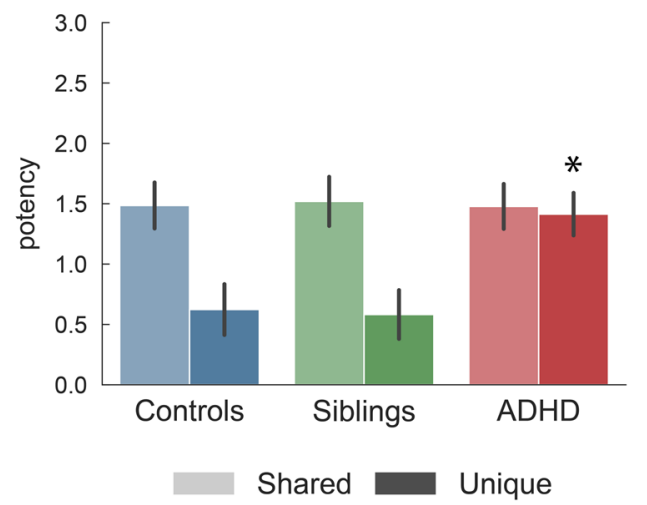

Fig. 4 Modulation of edges. The graphs quantify the average task potency across unique or shared connections for each group and connection type (corresponding to the third line in Fig. 1). All reported values show the average and standard deviation across 10,000 independent bootstraps. Indicated $P$ values show significant differences after FDR correction. Full results are available in Tables $\mathrm{S} 3$ and $\mathrm{S} 7$. Replication of these findings in light of possible confounding effects (scanner, gender, medication, comorbidity, age, IQ) is available in Figs. S1 and S2.

task switching more demanding, more 'expensive' and inefficient, with more challenging task performance as a result. As such, this connectivity profile provides support for the cognitive-energetic model of $\mathrm{ADHD}^{2}$. In this model, the limitations in arousal observed in ADHD could be a consequence of a higher level of energy required to perform cognitive tasks. This is potentially related to having to micro-manage $P_{\text {single }}$ patterns instead of keeping a general processing core ready to perform. Assessing individual-specificities of these task-tailored connectivity 
in protocols including multiple tasks and mind-wandering detection could help disentangle whether this connectivity profile reflects coping strategies from possible distracting thoughts during the task.

Fitting with the hypothesis of inefficient neural processing is the observation that participants with ADHD typically are able to perform most tasks but exhibit large variability in the way they perform tasks ${ }^{16,28,29}$. Some studies have reported an inefficient use of resources for specific networks or functions in ADHD including the attention network ${ }^{29,30}$, executive functioning ${ }^{6,31}$ or cognitive control ${ }^{29,32}$. However, as these studies focus on specific cognitive aspects, they do not allow identifying a potential task-general underlying deficit, as demonstrated in the present study. Alternative approaches to investigating the efficiency of the brain's organization have used graph theory and shown that the functional architecture of the ADHD brain is associated with differences in the balance of local and global efficiency ${ }^{7,33-35}$. However, these graph theory metrics provide no information on localized effects affecting specific cognitive functions. In contrast, our integrated approach provides a bridge between cognitive tasks and the functional architecture of the brain to understand the interaction between neural systems.

Unaffected siblings of individuals with ADHD share on average $50 \%$ of their genetic make-up with the ADHD probands, and accordingly, are hypothesized to share part of the ADHD endophenotype, i.e. biological deficits

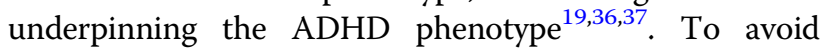
genetic relatedness between our groups, we selected unaffected siblings and ADHD from independent families that reduced our sample size but validate a biological endophenotype. Siblings displayed a task connectivity profile in between that of controls and ADHD participants. Siblings showed a (non-significantly) larger use of $P_{\text {single }}$ connections and some differences in the choice of $P_{\text {all }}$ connections compared with controls. In terms of localization of $P_{\text {all }}$ connections (see Fig. 3 and S6), they combined motor connectivity like controls and subcortical connectivity like ADHD, which enables to perform the task as well as controls and which might be a more efficient alternative strategy, as it requires less modulation of connectivity. The strongest differences are observed in the stop signal reaction time variability for ADHD participants. No differences in performance are observed between unaffected siblings of ADHD and the control participants (see Table S5). Previous research suggests that ADHD participants could compensate by using higher order executive systems or by relying more on lower-order visual, spatial and motoric processing ${ }^{38-41}$. Our results support the literature by suggesting that unaffected siblings are potentially still able to recruit more task-general efficient connections.
The results shown in Fig. 2 also highlight that $P_{\text {single }}$ connections can be used to investigate such compensatory mechanisms at the level of individual participants. $P_{\text {single }}$ connections are highly variable across participants, which is also described in previous work on task potency ${ }^{9}$. For instance, using longitudinal designs and models of compensatory strategies ${ }^{41}$, we can focus on those connections that are subject-specific and highly reproducible at the individual level to investigate a progressive specialization of individual compensatory mechanisms. In contrast to the high variability of $P_{\text {single }}$ connections, the absence of some $P_{\text {all }}$ connections was highly reproducible across ADHD and siblings compared with controls (Fig. 2). As such, an absence of $P_{\text {all }}$ connections may have potential as a biomarker for ADHD. This would encourage further, out-of-sample investigations of our new task potency approach.

Brain areas involved in the $P_{\text {all }}$ connections and the associated differences between ADHD and controls are shown in Fig. 3 and S6. At the brain regional level, participants with ADHD mainly missed modulations that connect regions within the executive control and reward pathways, including cerebellum, striatal, cingulum and cortical areas during task performance ${ }^{33}$. These results are coherent with meta-analysis showing hypoconnectivity in fronto-parieto-cingulo-striatal circuit ${ }^{6,26}$. As shown in Fig. 3 and S6, participants with ADHD preserved only few $P_{\text {all }}$ connections, interestingly involving striatal regions known to be involved in reward processing. Note that these results do not contradict typical findings of aberrant brain activity in reward-related regions in participants with $\mathrm{ADHD}^{5}$ as we showed that participants with ADHD used these connections with greater inconsistency and decreased modulation compared with controls. Knowing that ADHD participants make less efficient use of common pathways to govern multiple cognitive functions, will inform next studies aimed at understanding task response variability in ADHD.

Another important follow up is to integrate knowledge on resting state differences ${ }^{7}$. As the task modulation builds upon the baseline architecture, after identifying differences between groups during task processing, both levels of baseline and modulation need to be integrated. This will also allow an understanding of the dependency between baseline and task effect and whether task connectivity differences can be predicted on the basis of baseline alterations ${ }^{42}$. The choice of task in this study is aimed to target cognitive functions altered in ADHD and follow up studies need to extend this finding to other cognitive domains to frame the $P_{\text {all }}$ edges toward specific localized circuit or show a generalization of this effect. In addition, longitudinal studies need to assess age-related trajectories of these findings, as we know that task potency changes with age ${ }^{43}$. Performance on these tasks 
also changes with further maturation and changes in symptoms severity that can occur in ADHD with age. The current study does not address a possible developmental delay explanation ${ }^{44}$, as it would require a longitudinal database to be able to disentangle the complex interaction between symptom severity, age and performance in relation to differences in connectivity.

In conclusion, we examined connectivity modulations across three tasks and demonstrated that individuals with ADHD showed less $P_{\text {all }}$ and more $P_{\text {single }}$ connectivity modulations when compared with controls or unaffected siblings. Our work represents an important step towards new integrative theories explaining how multiple neural alterations interact and result into multiple cognitive impairments in ADHD. Future studies should explore whether the results hold under other tasks paradigms.

\section{Acknowledgements}

This work was supported by a Marie Curie International Incoming Fellowship under the European Union's Seventh Framework Programme (FP7/ 2007-2013), Grant 327340 (Brain Fingerprint) to MM; The Netherlands Organization for Scientific Research (NWO) Grant NWO-Vidi 864-12-003 to CFB and Wellcome Trust UK Strategic Award [098369/Z/12/Z] to CFB. Additional support was received from the European Community's Horizon 2020 Programme (H2020/2014-2020) under grant agreements 667302 (CoCA) and 728018 (Eat2beNICE). BF is supported by a personal Vici grant of the Netherlands Organization for Scientific Research (NWO; grant 016-130-669).

The NeurolMAGE study was supported by NIH Grant R01MH62873, NWO Large Investment Grant 1750102007010 (to JB), ZonMW grant 60-60600-97-193, NWO grants 056-13-015 and 433-09-242, and matching grants from Radboud University Medical Center, University Medical Center Groningen and Accare, and Vrije Universiteit Amsterdam. The research leading to these results also received support from the European Community's Seventh Framework Programme (FP7/2007-2013) under grant agreement number 278948 (TACTICS) and 603016 (MATRICS) and the Innovation Medicine Initiative grants 115300 (EU-AIMS) and 777394 (AIMS-2-TRIALS).

\section{Author details}

'Department of Cognitive Neuroscience, Radboud University Medical Center, Nijmegen, The Netherlands. ${ }^{2}$ Donders Institute for Brain, Cognition and Behaviour, Radboud University, Nijmegen, The Netherlands. ${ }^{3}$ Department of Neurology, Washington University School of Medicine, St Louis, USA. ${ }^{4}$ Karakter Child and Adolescent Psychiatry University Centre, Nijmegen, The Netherlands. ${ }^{5}$ School of Psychological Sciences, Monash Institute of Cognitive and Clinical Neurosciences, Monash University, Melbourne, VIC, Australia. ${ }^{6}$ Departments of Human Genetics and Psychiatry, Radboud University Medical Center, Nijmegen, The Netherlands. 'University of Groningen, University Medical Center Groningen, Department of Psychiatry, Groningen, The Netherlands. ${ }^{8}$ Amsterdam UMC, University of Amsterdam \& Vrije Universiteit Amsterdam, Emma Neuroscience Group at Emma Children's Hospital, department of Pediatrics, Amsterdam Reproduction \& Development, Amsterdam, The Netherlands. ${ }^{9}$ Clinical Neuropsychology section, Vrije Universiteit, Van der Boechortstraat 7, 1081 BT Amsterdam, The Netherlands. ${ }^{10}$ Centre for Functional MRI of the Brain (FMRIB), University of Oxford, Oxford, UK

\section{Code availability}

Scripts used to generate all analysis are available at: https://github.com/roscha/ task_potency/.

\section{Conflict of interest}

J.K.B. has been in the past 3 years a consultant to / member of advisory board of / and/or speaker for Shire, Roche, Medice, and Servier. He is not an employee of any of these companies, and not a stock shareholder of any of these companies. He has no other financial or material support, including expert testimony, patents, royalties. Barbara Franke has received educational speaking fees from Medice and Shire. R.J.C., M.O., C.H., D.J.H., P.J.H., J.O., C.F.B., M.M. reported no financial interests or potential conflicts of interest.

\section{Publisher's note}

Springer Nature remains neutral with regard to jurisdictional claims in published maps and institutional affiliations.

Supplementary information The online version contains supplementary material available at https://doi.org/10.1038/s41398-021-01284-z.

Received: 27 May 2020 Revised: 27 January 2021 Accepted: 19 February 2021

Published online: 10 March 2021

\section{References}

1. Faraone, S. V. et al. Attention-deficit/hyperactivity disorder. Nat. Rev. Dis. Prim. 1 (Aug), 15020 (2015).

2. Sergeant, J. The cognitive-energetic model: an empirical approach to attention-deficit hyperactivity disorder. Neurosci. Biobehav. Rev. 24(Jan), 7-12 (2000).

3. Barkley, R. A. Behavioral inhibition, sustained attention, and executive functions: constructing a unifying theory of ADHD. Psychol. Bull. 121(Jan), 65-94 (1997).

4. Sonuga-Barke, E. J. S. The dual pathway model of AD/HD: an elaboration of neuro-developmental characteristics. Neurosci. Biobehav Rev. 27(Nov), 593-604 (2003).

5. Luman, M., Oosterlaan, J. \& Sergeant, J. A. The impact of reinforcement contingencies on AD/HD: a review and theoretical appraisal. Clin. Psychol. Rev. 25(Feb), 183-213 (2005)

6. Cortese, S. The neurobiology and genetics of attention-deficit/hyperactivity disorder (ADHD): what every clinician should know. Eur. J. Paediatr. Neurol. 16 (Sep), 422-433 (2012).

7. Posner, J., Park, C. \& Wang, Z. Connecting the dots: a review of resting connectivity MRI studies in attention-deficit/hyperactivity disorder. Neuropsychol. Rev. 24(Mar), 3-15 (2014).

8. Purper-Ouakil, D., Ramoz, N., Lepagnol-Bestel, A.-M., Gorwood, P. \& Simonneau, M. Neurobiology of attention deficit/hyperactivity disorder. Pediatr. Res. 69 (May), 69R-76R (2011).

9. Chauvin, R. J., Mennes, M., Llera, A., Buitelaar, J. K. \& Beckmann, C. F. Disentangling common from specific processing across tasks using task potency. Neurolmage 184(Jan), 632-645 (2019).

10. Fox, M. D., Zhang, D., Snyder, A. Z. \& Raichle, M. E. The global signal and observed anticorrelated resting state brain networks. J. Neurophysiol. 101(Jun), 3270-3283 (2009).

11. Mennes, M., Kelly, C., Colcombe, S., Castellanos, F. X. \& Milham, M. P. The extrinsic and intrinsic functional architectures of the human brain are not equivalent. Cereb. Cortex. 23(Jan), 223-229 (2013).

12. Smith, S. M. et al. Correspondence of the brain's functional architecture during activation and rest. Proc. Natl Acad. Sci. U.S.A. 106(Aug), 13040-13045 (2009).

13. Fedorenko, E., Duncan, J. \& Kanwisher, N. Broad domain generality in focal regions of frontal and parietal cortex. Proc. Natl Acad. Sci. U.S.A. 110(Oct), 16616-16621 (2013)

14. Stiers, P., Mennes, M. \& Sunaert, S. Distributed task coding throughout the multiple demand network of the human frontal-insular cortex. Neuroimage $\mathbf{5 2}$ (Aug), 252-262 (2010).

15. Tavor, I. et al. Task-free MRI predicts individual differences in brain activity during task performance. Science 352(Apr), 216-220 (2016).

16. van Rooij, D. et al. Altered neural connectivity during response inhibition in adolescents with attention-deficit/hyperactivity disorder and their unaffected siblings. Neuroimage Clin. 7(Jan), 325-335 (2015).

17. von Rhein, D. et al. Increased neural responses to reward in adolescents and young adults with attention-deficit/hyperactivity disorder and their unaffected siblings. J. Am. Acad. Child Adolesc. Psychiatry 54(May), 394-402 (2015).

18. van Ewijk, H. et al. Visuospatial working memory in ADHD patients, unaffected siblings, and healthy controls. J. Atten. Disord. 18(May), 369-378 (2014).

19. Gottesman, I. I. \& Gould, T. D. The endophenotype concept in psychiatry: etymology and strategic intentions. Am. J. Psychiatry 160(Apr), 636-645 (2003).

20. Glahn, D. C. et al. Arguments for the sake of endophenotypes: examining common misconceptions about the use of endophenotypes in psychiatric 
genetics. Am. J. Med Genet. B Neuropsychiatr. Genet. 165B(Mar), 122-130 (2014).

21. von Rhein, D. et al. The NeurolMAGE study: a prospective phenotypic, cognitive, genetic and MRI study in children with attention-deficit/hyperactivity disorder. Design and descriptives. Eur. Child Adolesc. Psychiatry 24-3(Aug), 1-17 (2015).

22. van Oort, E. S. B et al. Functional parcellation using time courses of instantaneous connectivity. Neuroimage. 170(Apr) 31-40 (2018)

23. Feinberg, D. A. et al. Multiplexed echo planar imaging for sub-second whole brain FMRI and fast diffusion imaging. PLOS One. 5(Dec), e15710 (2010).

24. Bielczyk, N. Z. et al. Thresholding functional connectomes by means of mixture modeling. Neurolmage 171, 402-414 (2018)

25. Gupta, A. et al. Patterns of brain structural connectivity differentiate normal weight from overweight subjects. Neuroimage Clin. 7, 506-517 (2015).

26. Rubia, K. et al. Disorder-specific inferior prefrontal hypofunction in boys with pure attention-deficit/hyperactivity disorder compared to boys with pure conduct disorder during cognitive flexibility. Hum. Brain Mapp. 31(Dec), 1823-1833 (2010).

27. Nomi, J. S. et al. Chronnectomic patterns and neural flexibility underlie executive function. Neuroimage. 147(Feb), 861-871 (2017)

28. Tamm, L. et al. Reaction time variability in ADHD: a review. Neurotherapeutics $\mathbf{9}$ (Jul), 500-508 (2012).

29. Shiels, K. \& Hawk, L. W. Self-regulation in ADHD: the role of error processing. Clin. Psychol. Rev. 30(Dec), 951-961 (2010).

30. Booth, J. E., Carlson, C. L. \& Tucker, D. M. Performance on a neurocognitive measure of alerting differentiates ADHD combined and inattentive subtypes: a preliminary report. Arch. Clin. Neuropsychol. 22(May), 423-432 (2007).

31. Fuggetta, G. P. Impairment of executive functions in boys with attention deficit/hyperactivity disorder. Child Neuropsychol. 12(Feb), 1-21 (2006).

32. Randall, K. D., Brocki, K. C. \& Kerns, K. A. Cognitive control in children with ADHD-C: how efficient are they? Child Neuropsychol. 15(Mar), 163-178 (2009).
33. Konrad, K. \& Eickhoff, S. B. Is the ADHD brain wired differently? A review on structural and functional connectivity in attention deficit hyperactivity disorder. Hum. Brain Mapp. 31(Jun), 904-916 (2010).

34. Lin, P. et al. Global and local brain network reorganization in attention-deficit/ hyperactivity disorder. Brain Imaging Behav. 8(Dec), 558-569 (2014).

35. Wang, L. et al. Altered small-world brain functional networks in children with attention-deficit/hyperactivity disorder. Hum. Brain Mapp. 30(Feb), 638-649 (2009).

36. van Rooij, D. et al. Distinguishing adolescents with ADHD from their unaffected siblings and healthy comparison subjects by neural activation patterns during response inhibition. Am. J. Psychiatry 172(Jan), 674-683 (2015).

37. Wolfers, T. et al. Quantifying patterns of brain activity: distinguishing unaffected siblings from participants with ADHD and healthy individuals. NeuroImage: Clin. 12(Feb), 227-233 (2016).

38. Young, S. Coping strategies used by adults with ADHD. Personal. Individ. Differences. 38(Mar), 809-816 (2005).

39. Fassbender, C. \& Schweitzer, J. B. Is there evidence for neural compensation in attention deficit hyperactivity disorder? A review of the functional neuroimaging literature. Clin. Psychol. Rev. 26(Aug), 445-465 (2006).

40. Livingston, L. A. \& Happé, F. Conceptualising compensation in neurodevelopmental disorders: reflections from autism spectrum disorder. Neurosci. Biobehav. Rev. 80(Sep), 729-742 (2017).

41. Merkt, J., Reinelt, T. \& Petermann, F. A framework of psychological compensation in attention deficit hyperactivity disorder. Front Psychol. 6, 1580 (2015).

42. Ito, T. et al. Cognitive task information is transferred between brain regions via resting-state network topology. Nat Commun 8(Oct), 1027 (2017)

43. Chauvin, R. J. Mennes, M., Buitelaar, J. K. \& Beckmann, C. F. Assessing agedependent multi-task functional co-activation changes using measures of task-potency. Dev. Cogn. Neurosci. 33(Dec), 5-16 (2018)

44. Dyck, M. J. \& Piek, J. P. Developmental delays in children with ADHD. J. Atten Disord. 18(Jul), 466-478 (2014). 